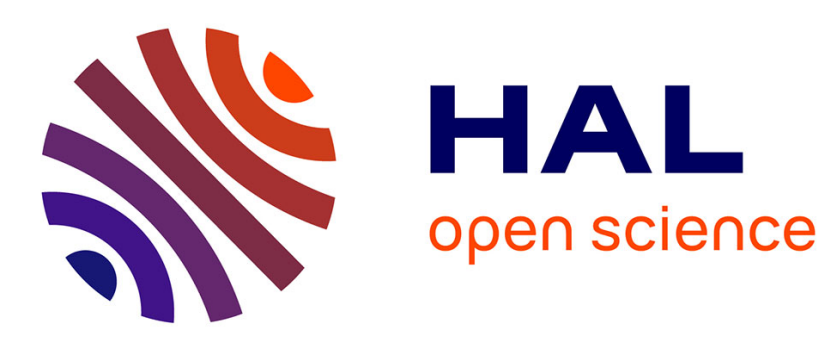

\title{
Characterization and Compensation of XY Micropositioning Robots using Vision and Pseudo-Periodic Encoded Patterns.
}

Ning Tan, Cédric Clévy, Guillaume J. Laurent, Patrick Sandoz, Nicolas Chaillet

\section{To cite this version:}

Ning Tan, Cédric Clévy, Guillaume J. Laurent, Patrick Sandoz, Nicolas Chaillet. Characterization and Compensation of XY Micropositioning Robots using Vision and Pseudo-Periodic Encoded Patterns.. IEEE International Conference on Robotics and Automation, ICRA'2014., Jan 2014, Hong Kong SAR China. pp.1-6. hal-01051712

\section{HAL Id: hal-01051712 \\ https://hal.science/hal-01051712}

Submitted on 25 Jul 2014

HAL is a multi-disciplinary open access archive for the deposit and dissemination of scientific research documents, whether they are published or not. The documents may come from teaching and research institutions in France or abroad, or from public or private research centers.
L'archive ouverte pluridisciplinaire HAL, est destinée au dépôt et à la diffusion de documents scientifiques de niveau recherche, publiés ou non, émanant des établissements d'enseignement et de recherche français ou étrangers, des laboratoires publics ou privés. 


\title{
Characterization and Compensation of XY Micropositioning Robots using Vision and Pseudo-Periodic Encoded Patterns
}

\author{
Ning Tan, Cédric Clévy, Guillaume J. Laurent, Patrick Sandoz and Nicolas Chaillet
}

\begin{abstract}
Accuracy is an important issue for microrobotic applications. High accuracy is usually a necessary condition for reliable system performance. However there are many sources of inaccuracy acting on the microrobotic systems. Characterization and compensation enable reduction of the systematic errors of the micropositioning stages and improve the positioning accuracy. In this paper, we propose a novel method based on vision and pseudo-periodic encoded patterns to characterize the position-dependent errors along XY stages. This method is particularly suitable for microscale motion characterization thanks to its high range-to-resolution ratio and avoidance of camera calibration. Based on look-up tables and interpolation techniques, we perform compensation and get improved accuracy. The experimental results show an accuracy improved by $84 \%$ for square tracking and by $68 \%$ for random points reaching (respectively from $22 \mu \mathrm{m}$ to $3.5 \mu \mathrm{m}$ and from $22 \mu \mathrm{m}$ to $7 \mu \mathrm{m})$.
\end{abstract}

\section{INTRODUCTION}

Micromanipulations, such as microassembly [1], biological micromanipulation [2], microdispensing [3] require highly reliable and accurate operations. Considering many factors (e.g., success rate, speed, and contamination), these tasks usually rely on microrobotic systems with automatic control instead of manual operation [5], [6]. Micromanipulation platforms usually consist of one or several microrobots comprising several micropositioning stages [7].

Off-the-shelf micropositioning stages have inherent imperfections that could be noticeable issues to achieve a micrometer accuracy. Manufacturers provide generally statistical specifications such as positioning repeatability and sensor resolution. Because some of imperfections are positiondependent, these data are not sufficient to ensure a good accuracy of an end-effector attached to the stage. For instance, for one of such stage mounted with a tip of $20 \mathrm{~mm}$ length, the error could be around $3 \mu \mathrm{m}$ at the end-point in the perpendicular direction of the motion due to yaw deviation [11].

Moreover, micropositioning stages usually have limited Degrees-of-Freedom (DoF) and the assembling of several of them is required to meet specific needs. Tools such as gripper or probe are also fastened onto the stages as endeffector. These assemblies introduces geometric errors that must be evaluated and compensated. For example, if the

These works have been funded by the Franche-Comté région and OSEO and partially supported by the Labex ACTION project ANR-11-LABX01-01 and by the French RENATECH network through its FEMTO-ST technological facility. We would like to acknowledge David Guibert for technical support.

Authors are with the FEMTO-ST Institute, UFC-ENSMM-UTBMCNRS UMR 6174, Université de Franche-Comté, Besançon 25000, France cedric.clevy@femto-st.fr perpendicularity error between two $\mathrm{X}$ and $\mathrm{Y}$ axes is $0.1^{\circ}$, $1 \mathrm{~cm}$ motion along $\mathrm{Y}$ could induce error about $17 \mu \mathrm{m}$ along $\mathrm{X}$ which is significant at the microscale.

To achieve motions of end-effector with improved accuracy, assembly errors and position-dependent errors must be measured, quantified and compensated. This job requires an exteroceptive sensor measuring the position of the endeffector of the micromanipulation platform. However, exteroceptive sensors that have at the same time nanometric resolutions, millimeter ranges of measurement and multiple direction of measurement are very rare [9].

For instance, position sensors such as interferometers that have a high range-to-resolution ratio are generally bulky and offer only one direction of measurement. Multi-direction of measurement requires to combine several sensors, which is a tough task because of the limited workspace and particularly because it is difficult to measure the position of the endeffector when it is moving in another direction than the measured one.

Vision is a rational alternative to measure the position of the end-effector in several directions. However developed methods such as blob detection [10], model-based tracking [21] and phase-like correlation methods [14] have a limited range of measurement because the object of interest has to remain inside the field-of-view. As a consequence, a trade-off must be done between range and resolution.

In this paper, we propose a novel method of characterization using vision and pseudo-periodic encoded patterns. A specific image processing enables high resolution and long ranges in the two directions of the image plane. We show on a case study that these features make pseudoperiodic encoded patterns the ideal candidate to characterize the motion behavior of microrobots. The case study is a XY microrobotic structure using two micropositioning stages because this kind of structure is very popular in microscale applications [12], [13]. The second contribution of the paper is the improving of the accuracy thanks to a compensated driving based on look-up tables and interpolation techniques.

The remainder of this paper is organized as follows. Section II introduces the visual measurement principle. Section III presents the experimental setup including the micropositioning stages and vision system. The errors characterization of the XY micropositioning robot is presented in Section IV. Section V details the compensation principle and experimental compensation results. Finally, we conclude the paper with Section VI. 


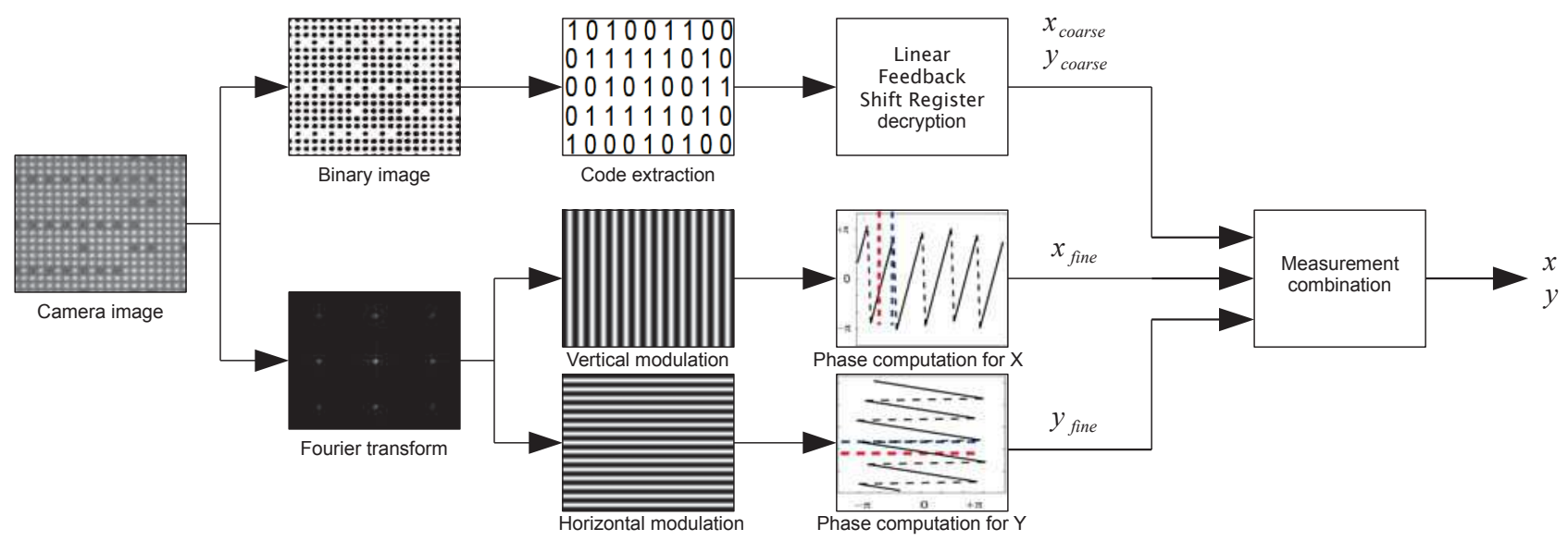

Fig. 1. Visual position measurement process (PPP algorithm).

\section{VISUAL POSITION MEASUREMENT USING PSEUDO-PERIODIC ENCODED PATTERNS}

Most of high-resolution imaged-based motion-detection algorithms rely on phase-like correlation methods. For example, this kind of method has been implemented by Moddemeijer [14] reporting a resolution of $13.3 \mathrm{~nm}$. The main drawback of these correlation-like methods remains in the useful field-of-view. Those methods are feature dependent so that the pattern has to remain inside the region of interest to be analyzed thus limiting the range of measurement. To overcome such a drawback, a technique based on pseudoperiodic patterns has been proposed by different authors [15], [16], [17].

In this paper, we propose to use a similar technique which is based on an encryption of a binary code over a pseudoperiodic pattern (PPP). The position is obtained by combining fine and coarse measurements that are complementary (cf. Fig. 1). The coding allows absolute but coarse coordinate transformations of the image reference frame into actual positions on the observed part of the pattern. In addition, the pseudo-periodic pattern allows a high level of interpolation through phase measurements that lead to subpixel resolution.

The coarse measurement is done by decrypting the distribution of points missing in the periodic frame. Some dots are missing and their distribution follows a codification of the $\mathrm{X}$ and Y orders of the dots. This codification is based on Linear Feedback Register Sequences (LFRS). Pose retrieval involves complementary image processing to identify the location of the missing points and thus to return the line and column orders necessary to complete the fine position provided by phase computations.

The fine measurement is performed after a 2D Fourier transformation that aims to separate the different directions of modulation of the pattern. The phase of the periodic grid is then computed in both directions thanks to two analysis functions. Given the phases (in rad) and the period of the pattern (in meter), it is straightforward to calculate the relative position of the pattern in the image reference (a)

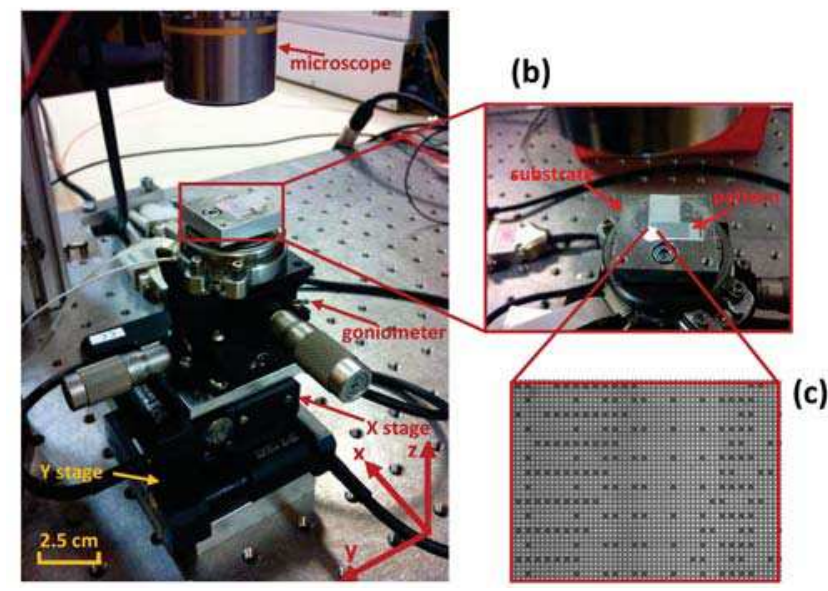

Fig. 2. Experimental setup of the $\mathrm{XY} \Theta$ microrobotic system, (a) general view, (b) close view of the end-plateform, (c) microscope image of the pattern.

frame. This process gives the position with typically subpixel resolution of $10^{-3}$ pixels but also with an indeterminacy equal to the wavelength of the pattern.

Moreover as the period of the pattern is precisely known (4 $\mu \mathrm{m}$ in the present), the measurement is intrinsically selfcalibrated. There is no need to calibrate the imaging system. More details about the algorithm and the fabrication of the pattern can be found in [18].

Finally, in the present case, the measuring range is limited by the size of the pattern that is $9.5 \mathrm{~mm}$ for $\mathrm{x}$-axis and $4.2 \mathrm{~mm}$ for $y$-axis. The reproducibility of the visual measurement has been experimentally evaluated and is better than $10 \mathrm{~nm}$ [19].

\section{CASE STUDY}

Many micromanipulation systems work with mobile parts that are guided based on friction principles. Their positioning performances depend on the qualities of fabrication, plays, 
TABLE I

SPECIFICATIONS OF XY TRANSLATION STAGES IN DATASHEET

\begin{tabular}{lc}
\hline Stage & PI M-111.1DG \\
\hline Travel range & $15 \mathrm{~mm}$ \\
Resolution & $50 \mathrm{~nm}$ \\
Unidirectional repeatability & $100 \mathrm{~nm}$ \\
Pitch angle deviation & $\pm 150 \mu \mathrm{rad}$ \\
Yaw angle deviation & $\pm 150 \mu \mathrm{rad}$ \\
Backlash & $2 \mu \mathrm{m}$ \\
Thread pitch & $0.4 \mathrm{~mm}$ \\
Driving mechanism & Leadscrew \\
\hline
\end{tabular}

weight of the axes and so on. The micropositioning stages are equipped with internal sensors and are individually closedloop controlled in actuation layer. But depending of the location the sensors in the actuation chain, the feedback control can not reject some sources of errors. Moreover, assemblies errors can not be compensated using only proprioceptive sensors.

In this paper, we choose an XY microrobotic structure as a case study because this kind of structure is representative for many systems commonly used in micromanipulation. The pictures of the whole experimental setup and the endplatform are shown in Fig. 2 (a) and (b). The system consisting of two translation stages (XY) is mounted on a anti-vibration table. The two translation stages are PI M111.1DG equipped with MercuryTM C-863 controllers. The specifications of translation stages $X Y$ from the datasheets is given in Table I.

The external measuring system for characterization consists of a $1024 \times 768$ video camera (AVT STINGRAY F125C), a microscope lens (Optem zoom 70XL), an objective with $10 \times$ magnification and the pseudo-periodic pattern (Fig. 2 (c)). The upper goniometer (M-GON40-U) and lower goniometer (M-GON40-L) are used for adjusting the parallelism between the pattern and the camera.

\section{CHARACTERIZATION OF POSITION-DEPENDENT ERRORS}

The position-dependent errors along axes are significant characteristics of the precise positioning stages. These errors are due to the geometric nature of the axes. For macroscale robotics, this type of errors is usually neglected in calibration which mainly focuses on kinematic parameters identification or elastic deformation. However, these errors become significant at the microscale, especially for Cartesian microrobots. The error curves are functions of axis coordinates, and the functions are different from one axis to another, so measurements of these errors for every axis are necessary. The position-dependent errors are calculated by comparing the measured positions (estimation of real positions) with the targeted positions (positions to be reached). The designed trajectories of measurement are 1-DoF straight lines, that is, one stage is moving, while another stage is kept static.

As depicted in Fig. 3, each of the micropositioning stage is controlled to reach appointed target coordinate $x_{T}, y_{T}$. The

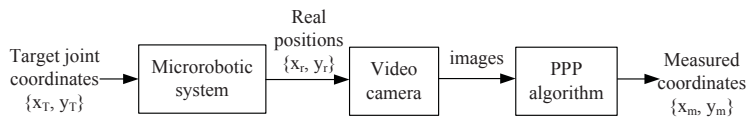

Fig. 3. Block diagram of characterization of position-dependent errors.

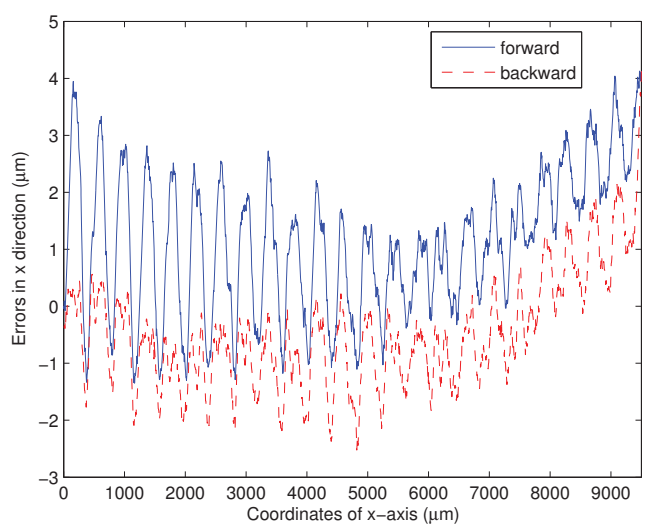

Fig. 4. Errors $\left(x_{T}-x_{m}\right)$ in $\mathrm{x}$ direction when $\mathrm{X}$ stage is moving forwards and backwards in one cycle.

camera captures the images of the pattern in the real positions $x_{r}, y_{r}$. The images are processed subsequently with the PPP algorithm so as to obtain measured coordinates $x_{m}$ and $y_{m}$. In the 9500 and $4200 \mu \mathrm{m}$ strokes, measurements are taken with $5 \mu \mathrm{m}$ as step size and a total of 11403 data in X direction and 5043 data in $\mathrm{Y}$ direction are obtained in 3 cycles (every cycle corresponds to one forward and one backward motion). Time spent on data acquisition is 9.5 hours.

According to the specification, the driving mechanism of the stage is leadscrew. So the errors along the axis could be foreseen somehow based on the mechanical property. The errors between $x_{T}$ and $x_{m}$ measured in one cycle is shown in Fig. 4. It can be seen that errors vary cyclically. Such behavior is reasonably assumed due to systematic turn-toturn nature inherent in the leadscrew. The thread pitch of the stage is $400 \mu \mathrm{m}$, so the cyclical error repeats with the same period. We can also see that the periods of the errors in forward and backward motions of the $\mathrm{X}$ stage are the same, but the magnitudes are slightly different. The driving system does not work symmetrically and makes a systematic error between forward and backward motions that corresponds to the backlash of $2 \mu \mathrm{m}$ as specified by the manufacturer.

Position-dependent errors appear not only in the driving direction but also in the lateral direction. Fig. 5 shows this coupling errors in y direction when only $\mathrm{X}$ stage is moving forwards and backwards. It can be seen that the coupling errors have the same period as the errors in driving direction.

Based on the characterized errors of all discrete targets along each single axis, we have enough information for all coordinates in 2-dimensional space.

We also used these measurements to calculate the positioning repeatability following ISO standard 9283 [20]. The objective is to get a lower bound of the accuracy 


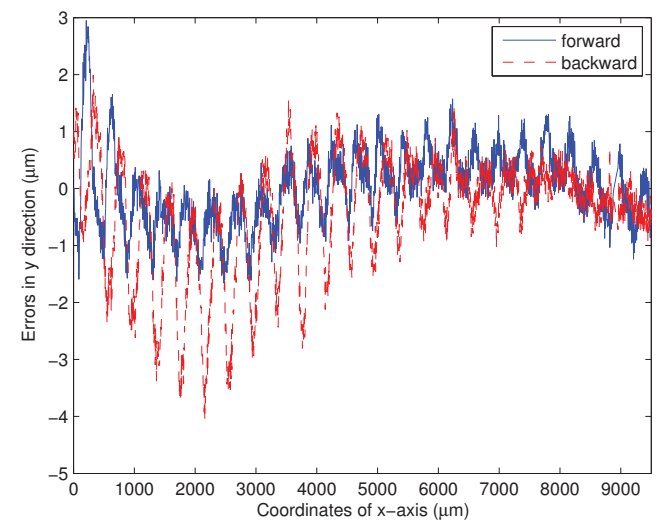

Fig. 5. Coupling errors $\left(0-y_{m}\right)$ in y direction when $\mathrm{X}$ stage is moving forwards and backwards in one cycle.

we could obtain with compensated driving. The positioning repeatability is defined by,

$$
R P=\bar{l}+3 S_{n-1}
$$

with,

$$
\bar{l}=\frac{1}{n} \sum_{j=1}^{n} l_{j}
$$

and,

$$
S_{n-1}=\sqrt{\frac{1}{n-1} \sum_{j=1}^{n}\left(l_{j}-\bar{l}\right)^{2}}
$$

$\bar{x}_{m}$ and $\bar{y}_{m}$ are the coordinates of the barycentre defined by $\bar{x}_{m}=\frac{1}{n} \sum_{j=1}^{n} x_{m, j}$ and $\bar{y}_{m}=\frac{1}{n} \sum_{j=1}^{n} y_{m, j} . l_{j}$ is the distance of the $j$ th measure to the barycentre $l_{j}=$ $\sqrt{\left(x_{m, j}-\bar{x}\right)^{2}+\left(y_{m, j}-\bar{y}\right)^{2}}$.

Fig. 6 shows the obtained repeatability along $\mathrm{x}$ for all measuring points of $\mathrm{X}$ axis. The curve for $\mathrm{Y}$ direction is similar. The repeatability is $\leq 1 \mu \mathrm{m}$ for most of the measuring points except for two peaks. This result is larger than expected from Table I. This is because of different experimental conditions between our configuration and that of specification evaluation by the constructor. The performance tests of PI company are indeed performed with less points and maybe with a more precise temperature control. For our case the ambient temperature drift was about $0.5^{\circ} \mathrm{C}$ for 9.5 hours. The conclusion of this calculation is that the residual errors even after the best compensation should theoretically not be better than this measured repeatability.

\section{COMPENSATION OF POSITION-DEPENDENT ERRORS}

\section{A. Compensation Principle}

As mentioned before, we have characterized the positiondependent errors of discrete coordinates along each axis. The error at a given point contains two parts, the first part induced by $X$ motion and the second part by $Y$ motion. We define

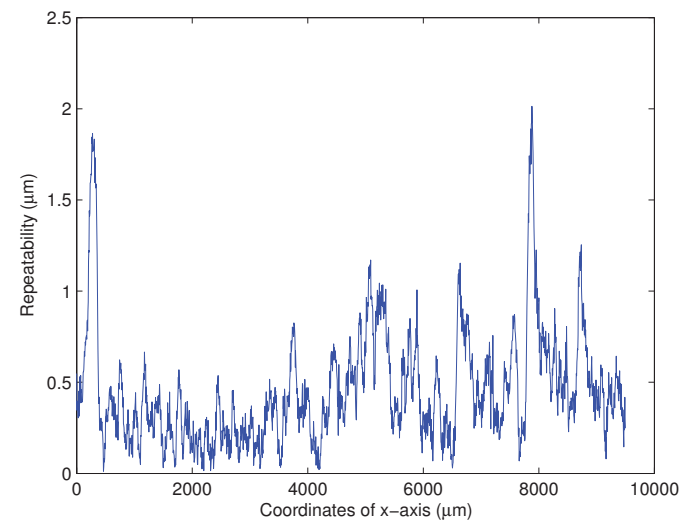

Fig. 6. Positioning repeatability in $x$ direction (forward motion).

$f_{x i}\left(x_{T}\right)$ and $f_{y i}\left(y_{T}\right)$ that denotes the errors component in $i$ direction when moving forward along $x$ or $y$ axis to target $x_{T}$ or $y_{T}$, and $b_{x i}\left(x_{T}\right)$ and $b_{y i}\left(y_{T}\right)$ that denotes the errors component in $i$ direction when moving backward along $x$ or $y$ axis to target $x_{T}$ or $y_{T}$. Each of these functions are based on a look-up table that records the previous measurements.

Hence, targets might not be in the look-up table, the errors at these targets neither. In this case, interpolation techniques are required to calculate the unknown errors (interpolated errors) based on the knowledge of known errors (characterized errors). We used a linear interpolation thanks to the interp $1 \mathrm{Matlab}^{\circledR}$ function. Cubic spline interpolation does not give significant better results.

The schematic diagram (Fig. 7) shows the compensation mechanism using the lookup table. Depending on the motion direction (forward or backward) and the target coordinates, the error components have various combinations. Taking $\mathrm{X}$ stage as an example, first the $k t h$ target position $x_{T k}$ and its previous target $x_{T k-1}$ are compared to identify the direction of the the motion (forward or backward); secondly, the program selects the corresponding errors from the lookup table of $\mathrm{X}$ stage based on the information of motion direction; finally these error components are summed up to form the total errors. The aggregated errors $\operatorname{ex}\left(x_{T}, y_{T}\right)$ and $e y\left(x_{T}, y_{T}\right)$ along $\mathrm{x}$ and along $\mathrm{y}$ are expressed by the following equations:

$$
\begin{gathered}
\operatorname{ex}\left(x_{T}, y_{T}\right)=\left[\begin{array}{c}
f_{x x}\left(x_{T}\right) \\
o r \\
b_{x x}\left(x_{T}\right)
\end{array}\right]+\delta_{x}\left(y_{T}\right)+\left[\begin{array}{c}
f_{y x}\left(y_{T}\right) \\
o r \\
b_{y x}\left(y_{T}\right)
\end{array}\right], \\
e y\left(x_{T}, y_{T}\right)=\left[\begin{array}{c}
f_{x y}\left(x_{T}\right) \\
o r \\
b_{x y}\left(x_{T}\right)
\end{array}\right]+\left[\begin{array}{c}
f_{y y}\left(y_{T}\right) \\
o r \\
b_{y y}\left(y_{T}\right)
\end{array}\right] .
\end{gathered}
$$

Due to the fact that the $\mathrm{Y}$ stage is not perfectly perpendicular to the $\mathrm{X}$ stage, the errors in $\mathrm{x}$ direction should be added by a bias $\delta_{x}\left(y_{T}\right)$ depending on the y coordinate.

The compensation principle relies on that the errors at the target points should be eliminated by adding the same amplitude of errors to the input. The input of the XY stages is 


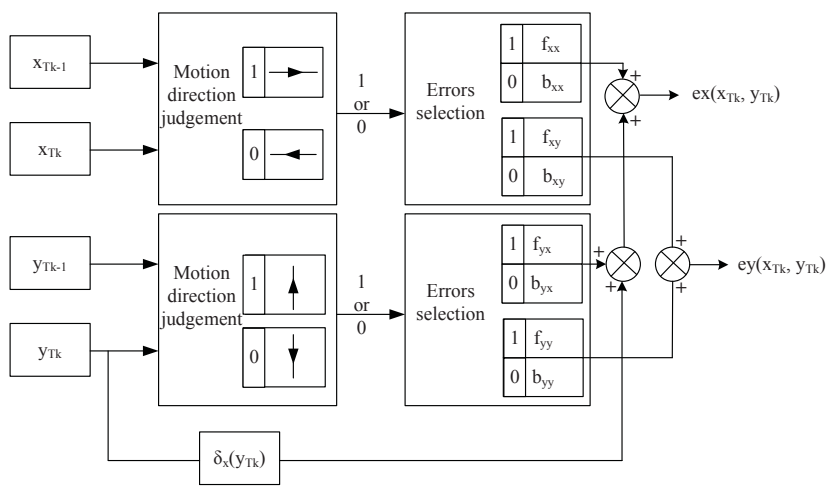

Fig. 7. Schematic diagram of the errors combination mechanism for compensation.

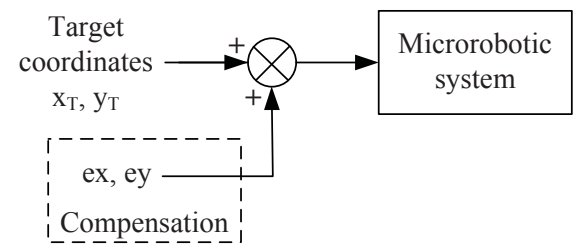

Fig. 8. Block diagram of compensation for position-dependent errors using lookup table.

then defined by the target trajectory minus the corresponding geometric errors in the lookup table instead of the target trajectory alone. The block diagram of the compensation process is shown in Fig. 8.

\section{B. Trajectories Tracking}

The first test is tracking of a square trajectory with compensation of position-dependent errors using lookup table. The planning trajectory is chosen with a size of 4000 $\times 4000 \mu \mathrm{m}$ square spanning from coordinates $10 \mu \mathrm{m}$ to $4010 \mu \mathrm{m}$. The square is divided into 4 segments: $\mathrm{AB}, \mathrm{BC}$, $\mathrm{CD}$, and DA (Fig. 9). The tracking performances with and without ( $e x=e x y=0$ ) compensation are shown in Fig. 10 (a) and (b). The accuracy (calculated following ISO standard 9283 [20]) is defined by:

$$
A P=\sqrt{\left(\bar{x}_{m}-x_{T}\right)^{2}+\left(\bar{y}_{m}-y_{T}\right)^{2}}
$$

Accuracy is about $22 \mu \mathrm{m}$ without compensation which is mainly due to the perpendicularity error (BC and DA segments) and a little bit position-dependent error ( $\mathrm{AB}$ and CD segments). After compensation, accuracy improves to approximately $4 \mu \mathrm{m}$ ( $84 \%$ error reduction).

\section{Random Points Positioning}

To examine the positioning accuracy of arbitrary points in the whole joint workspace experimentally, XY stages are commanded to reach random coordinates. The input targets $P_{T i}$ are coordinates of ten points which are generated randomly. Table II shows the coordinates of the ten targets defined for the test. The XY stages are controlled to reach the target $P_{T 1}, \ldots, P_{T 10}$ in sequence. From Fig. 11, we can

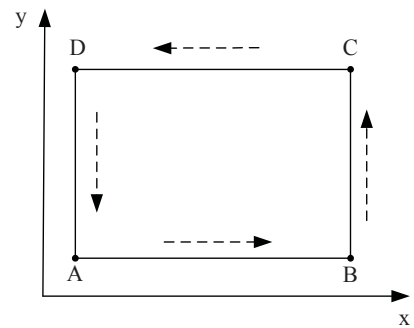

Fig. 9. Square trajectory (4 segments).

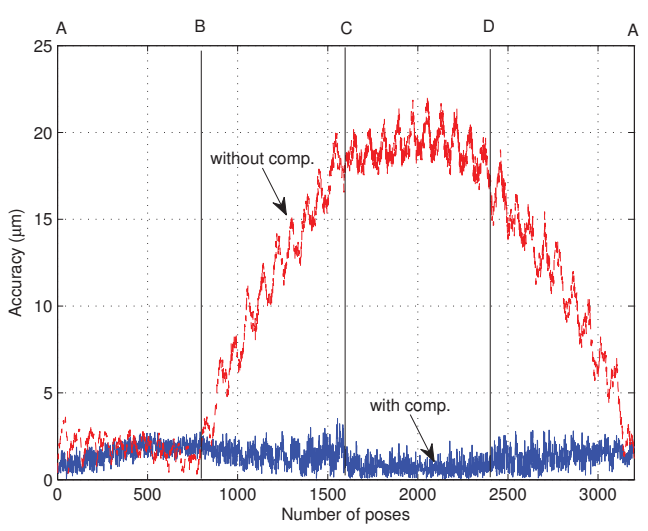

(a) Accuracy in all poses of tracking a square

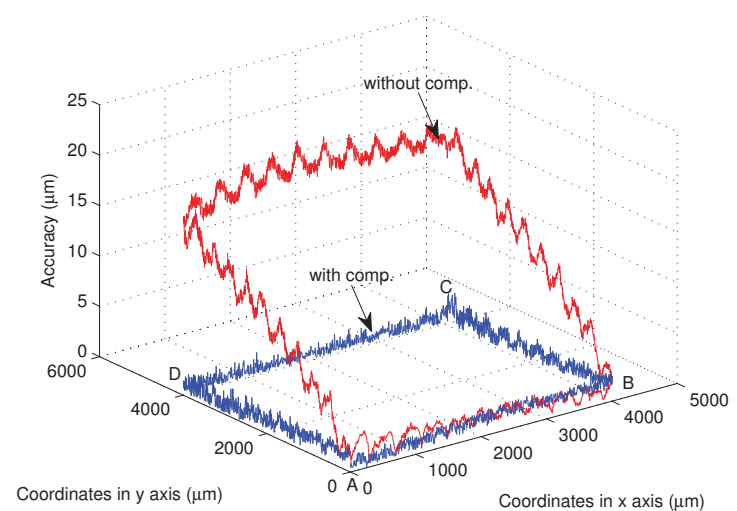

(b) 3D display of accuracy

Fig. 10. Accuracy of tracking square with and without compensation.

see that the positioning accuracy is improved from about $22 \mu \mathrm{m}$ to $7 \mu \mathrm{m}(68 \%$ reduction) by using the interpolated compensation.

\section{CONCLUSIONS}

Micropositioning stages are common components of the microrobotic systems for applications at the microscale. To achieve micrometer accuracy, errors in micropositioning stages required to be compensated. In this paper, we employed an novel measuring system consisting of a regular vision system observing a pseudo-periodic encoded pattern to measure the motion behavior of the XY micropositioning stages. This method is particularly suitable for microscale 
TABLE II

TABLE OF RANDOM TARGET COORDINATES

\begin{tabular}{ccc}
\hline Targets & $x_{T}(\mu m)$ & $y_{T}(\mu m)$ \\
\hline$P_{T 1}$ & 3471 & 2311 \\
$P_{T 2}$ & 6044 & 3991 \\
$P_{T 3}$ & 3673 & 39 \\
$P_{T 4}$ & 5118 & 2738 \\
$P_{T 5}$ & 3643 & 2071 \\
$P_{T 6}$ & 8196 & 186 \\
$P_{T 7}$ & 1702 & 756 \\
$P_{T 8}$ & 3776 & 3815 \\
$P_{T 9}$ & 3903 & 1390 \\
$P_{T 10}$ & 1035 & 35 \\
\hline
\end{tabular}

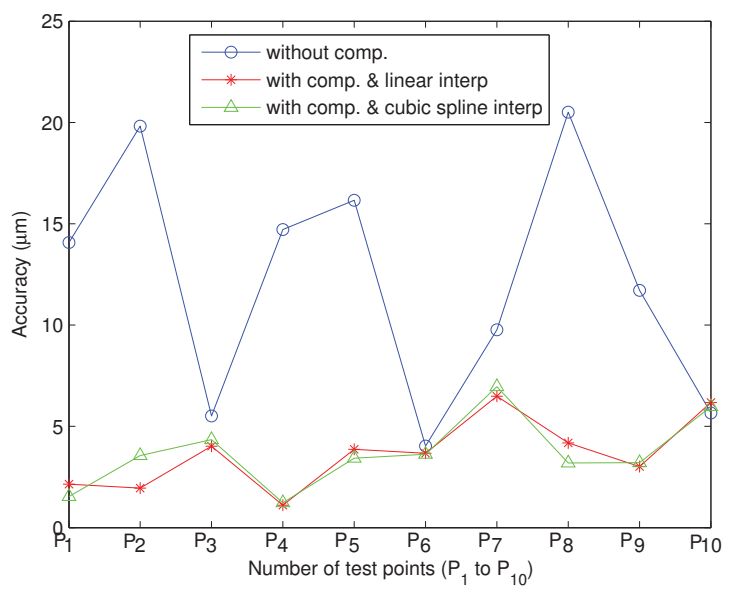

Fig. 11. Positioning accuracy of random points with and without compensation

motion characterization thanks to its high range-to-resolution ratio and avoidance of camera calibration.

We characterized the position-dependent errors along XY axes and perpendicularity error between them. Based on the quantified errors, compensation is conducted through building lookup tables and using linear interpolation. The experimental validations of the compensation show noticeable accuracy improvements despite the limited number of training points. The positioning accuracy is improved from $22 \mu \mathrm{m}$ to $3.5 \mu \mathrm{m}$ ( $84 \%$ reduction of inaccuracy) for square tracking and from $22 \mu \mathrm{m}$ to $7 \mu \mathrm{m}$ (68\% reduction of inaccuracy) for random points reaching.

Beyond these experimental results on a XY structure, the proposed method can be applied to other kind of microrobotic systems even having more degrees of freedom. The PPP algorithm can be extended to measure the angular position and also the out-of-plane motion ( $z$ direction) using depth-from-focus approach [17].

\section{REFERENCES}

[1] K. Rabenorosoa, C. Clévy, and P. Lutz "Active force control for robotic micro-assembly: application to guiding tasks," in IEEE International Conference on Robotics and Automation, Anchorage, AK, USA, 2010
[2] L. S. Mattos and D. G. Caldwell, "A fast and precise micropipette positioning system based on continuous camera-robot recalibration and visual servoing," in IEEE International Conference on Automation Science and Engineering, Bangalore, India, 2009.

[3] E. J. Griffith and S. Akella, "Coordinating multiple droplets in planar array digital microfluidic systems," International Journal of Robotics Research, vol. 24, no. 11, pp. 933-949, 2005.

[4] W. Park, C. R. Midgett, D. R. Madden, and G. S. Chirikjian, "A stochastic kinematic model of class averaging in single-particle electron microscopy," International Journal of Robotics Research, vol. 30, no. 6 , pp. 730-754, 2011.

[5] N. Chaillet and S. Régnier, Microrobotics for Micromanipulation. Wiley-ISTE, 2010.

[6] K. Rabenorosoa, C. Clévy, Q. Chen, and P. Lutz, "Study of forces during micro-assembly tasks using two-sensing-fingers gripper," IEEE/ASME Transactions on Mechatronics, vol. 17, no. 5, pp. 811$821,2012$.

[7] A. N. Das, R. Murthy, D. O. Popa, and H. E. Stephanou, "A multiscale assembly and packaging system for manufacturing of complex micro-nano devices," IEEE Transactions on Automation Science and Engineering, vol. 9, no. 1, pp. 160-170, 2012.

[8] D. O. Popa, R. Murthy, and A. N. Das, "M3-deterministic, multiscale, multirobot platform for microsystems packaging: design and quasistatic precision evaluation," IEEE Transactions on Automation Science and Engineering, vol. 6, no. 2, pp. 345-361, 2009.

[9] C. Clévy, M. Rakotondrabe, and N. Chaillet, Signal Measurement and Estimation Techniques for Micro and Nanotechnology. Springer, 2011.

[10] N. Tan, C. Clévy, G. J. Laurent, and N. Chaillet, "Calibration and validation of $\mathrm{XY} \Theta$ micropositioners with vision," in IEEE/ASME International Conference on Advanced Intelligent Mechatronics, Kachsiung, Taiwan, 2012.

[11] N. Tan, C. Clévy, and N. Chaillet, "Calibration of single-axis nanopositioning cell subjected to thermal disturbance," in IEEE International Conference on Robotics and Automation, Karlsruhe, Germany, 2013.

[12] Q. $\mathrm{Xu}, \mathrm{Y}$. $\mathrm{Li}$, and N. Xi, "Design, fabrication, and visual servo control of an XY parallel micromanipulator with piezo-actuation," IEEE Transactions on Automation Science and Engineering, vol. 6, no. 4, pp. 710-719, 2009.

[13] Y. K. Yong, S. S. Aphale, and S. O. Reza Moheimani, "Design, identification, and control of a flexure-based XY stage for fast nanoscale positioning," IEEE Transactions on Nanotechnology, vol. 8, no. 1, pp. 46-54, 2009.

[14] R. Moddemeijer, "On the determination of the position of extrema of sampled correlators", Signal Processing, IEEE Transactions on, vol. 39, no. 1, pp 216-219, 1991.

[15] P. Masa, E. Franzi, C. Urban, "Nanometric resolution absolute position encoders", CSEM Scientific and Technical Report, pp. 1-3, 2008.

[16] D.B. Boyton, "Position encoder using statistically biased pseudorandom sequence", US Patent App. 10/399, 470, april 18 ${ }^{t h}, 2003$.

[17] P. Sandoz, R. Zeggari, L. Froehly, J.L. Prétet, C. Mougin, "Position referencing in optical microscopy thanks to sample holders with outof-focus encoded patterns", Journal of Microscopy, vol. 225, pp. 293303, 2007.

[18] J.A. Galeano-Zea, P. Sandoz, E. Gaiffe, J.L. Prétet, C. Mougin, C., "Pseudo-Periodic Encryption of Extended 2-D Surfaces for High Accurate Recovery of any Random Zone by Vision", International Journal of Optomechatronics, vol. 4, no. 1, pp. 65-82, 2010.

[19] J.A. Galeano-Zea, P. Sandoz, E. Gaiffe, S. Launay, L. Robert, M. Jacquot, F. Hirchaud, J.L. Prétet, C. Mougin, "Position-referenced microscopy for live cell culture monitoring", Biomedical optics express, vol. 2, no. 5, 1307-1318,2011.

[20] Manipulating Industrial Robots Performance Criteria and Related Test Methods, ISO 9283, 1998.

[21] B. Tamadazte, S. Dembélé and N. Le Fort-Piat, "CAD model-based tracking and 3D visual-based control for MEMS microassembly", International Journal of Robotics Research, 29(11):1416-1434, 2010. 